\title{
IQC Synthesis based on Inertia Constraints
}

\author{
Anders Helmerssonn \\ Department of Electrical Engineering \\ Linköping University, SE-581 83 Linköping, Sweden \\ www: http://www. control.isy.liu.se \\ email: andersh@isy.liu.se
}

LiTH-ISY-R-1994

January 12, 1998

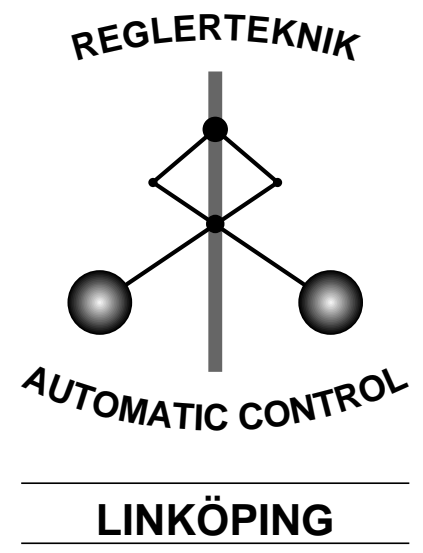

Technical reports from the Automatic Control group in Linköping are available as UNIX-compressed Postscript files by anonymous ftp at the address 130.236.20.24 (ftp.control.isy.liu.se). 


\title{
IQC Synthesis based on Inertia Constraints*
}

\author{
Anders Helmersson \\ Department of Electrical Engineering \\ Linköping University \\ SE-581 83 Linköping, Sweden \\ www: http://www.control.isy.liu.se \\ email: andersh@isy.liu.se
}

Submitted to Automatica

December 19, 1997

\begin{abstract}
Integral quadratic constraints (IQCs) can be used for proving stability of systems with uncertainties and nonlinearities. Similarly, IQCs can also be used for controller synthesis. Necessary and sufficient conditions for the existence of such a controller is derived. These conditions include linear matrix inequalities (LMIs) and matrix inertia specifying the number of negative eigenvalues of a matrix. In general, these conditions are nonconvex. Connections to bilinear matrix inequalities and LMIs with rank constraints are also given.

Keywords: controller synthesis, matrix inertia, linear matrix inequalities, integral quadratic constraints.
\end{abstract}

\section{Introduction}

Linear matrix inequalities (LMIs) have been used during the last ten years for analysis and synthesis of robust control systems. The reason for this emerging interest is twofold. First, analysis and synthesis problems can be formulated as LMIs. Secondly, efficient numerical solvers have been developed and are now available. One important feature of the LMI is that it defines a convex problem, for which the local solution (minimum) is also a global one.

However, some important problems, such as model reduction and synthesis of reduced order-controllers, cannot be formulated as pure LMIs. Instead, nonconvex elements, such as rank constraints or bilinear matrix inequalities (BMIs) must be included. In this paper we propose a new formulation based on the inertia of symmetric matrices, that is, the numbers of positive, negative and zero eigenvalues.

The LMI formulation can be derived from $\mathcal{H}_{\infty}$ and $\mu$ analysis. A more general analysis setting, based on integral quadratic constraints (IQCs) was

\footnotetext{
${ }^{*}$ This work was supported by the Swedish National Board for Industrial and Technical Development (NUTEK), which is gratefully acknowledged
} 
originally introduced by Yakubovich and later refined by Megretski and Rantzer [9]. In an IQC setting (after applying the Kalman-Yakubovich-Popov lemma) the synthesis problem can be formulated as an algebraic problem. Find a $K$ such that

$$
\left[\begin{array}{c}
A+B K C \\
I
\end{array}\right]^{*} \Pi\left[\begin{array}{c}
A+B K C \\
I
\end{array}\right]<0
$$

holds for some

$$
\Pi=\left[\begin{array}{cc}
P & \Gamma \\
\Gamma^{*} & -Q
\end{array}\right]
$$

defined to belong to a given convex set. The synthesis problem considers the condition on $\Pi, A, B$ and $C$ for the existence of such a solution $K$. Specifically, if $A, B$ and $C$ are given we want to search for such a $\Pi$.

Assuming that $\Pi$ is nonsingular, two LMIs can be derived: one in $\Pi$ and the other in $\Pi^{-1}$. This is in general not a convex problem. However, employing the inherent structure of some important problems, such as $\mathcal{H}_{\infty}$ and gain-scheduling synthesis, convexity can be recovered and the existence of a controller $K$ can be formulated as a (convex) LMI problem.

For the general synthesis problem, there seems to be no convex characterization of the existence of a controller $K$. One important class of problems that the convexity is violated in is synthesis of controller with a specified (low) order and model reduction. One way to solve this type of problem is by iterative projection methods [6]; using bilinear inequalities (BMIs) is another approach $[5,4,3,13]$.

Previous IQC synthesis results $[10,14,15]$ require $P$ and $Q$ in $(2)$ to be positive definite or at least positive semidefinite. These requirements are relaxed in this paper to inertia constraints on $\Pi$ only. In some IQC problems, the definiteness on $P$ and $Q$ must be relaxed in order to not produce too conservative results, see for instance [7].

In this paper we elaborate on general conditions for the existence of a controller $K$. Two conditions emerge: one LMI and one inertia constraint. The latter gives a constraint on the number of negative eigenvalues of a matrix that depends affinely on $\Pi$.

Section 2 gives a brief introduction of integral quadratic constraints (IQCs). In section 3 some basic facts on inertia of matrices are given. The main synthesis results are stated and proved in section 4 . Conclusions are given in section 5 .

\section{$1.1 \quad$ Notations}

Here $A^{*}$ denotes the (complex conjugate) transpose; $A^{\dagger}$ is the pseudo-inverse; $I_{n}$ denotes a unitary matrix of size $n \times n ; \nu(A)$ and $\pi(A)$ are the number of negative and positive eigenvalues of $A ; A^{\perp}$ denotes any full rank matrix such that $\operatorname{ker} A=\operatorname{range} A^{\perp}$, where $\operatorname{ker} A$ is the null space of $A$ and $\operatorname{range} A$ is the range or image of $A$. Note that $A^{\perp}$ exists only if $A$ has linearly dependent rows and that $A^{\perp} A=0$.

For a matrix

$$
M=\left[\begin{array}{ll}
M_{11} & M_{12} \\
M_{21} & M_{22}
\end{array}\right]
$$




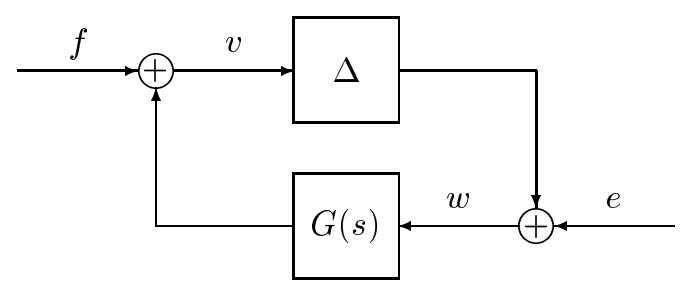

Figure 1: Basic feedback configuration.

and a matrix $Q$ with compatible dimension the lower fractional transformation (LFT) is defined as

$$
M \star Q=\mathcal{F}_{l}(M, Q)=M_{11}+M_{12} Q\left(I-M_{22} Q\right)^{-1} M_{21} .
$$

The set of rational stable transfer functions is denoted by $\mathcal{R} \mathcal{H}_{\infty}$ and $\mathcal{L}_{2}$ denotes the Lebesgue space of signals with bounded energy; $\mathcal{L}_{2 e}$ denotes the extended Lebesgue space of signals with bounded energy over a finite interval $[0, T]$.

\section{Integral Quadratic Constraints}

The integral quadratic constraints (IQCs) have been proposed for robustness analysis [9]. The IQC forms a stability criterion for the interconnection of a stable system $G \in \mathcal{R H}_{\infty}$ and a bounded causal operator $\Delta$, see figure 1 .

$$
\left\{\begin{array}{l}
v=G w+f \\
w=\Delta v+e .
\end{array}\right.
$$

We say that the interconnection of $G$ and $\Delta$ is well-posed if the map $(v, w) \rightarrow$ $(e, f)$ defined by $(3)$ has a causal inverse on $\mathcal{L}_{2 e}$. The interconnection is stable if, in addition, the inverse is bounded, that is, if there exists a constant $C$ such that

$$
\int_{0}^{T}\left(|v(t)|^{2}+|w(t)|^{2}\right) d t \leq C \int_{0}^{T}\left(|f(t)|^{2}+|e(t)|^{2}\right) d t
$$

for any $T \geq 0$ and for any solution of (3).

Depending on the particular application, various versions of IQCs are available. Two signals $w \in \mathcal{L}_{2}[0, \infty)$ and $v \in \mathcal{L}_{2}[0, \infty)$ are said to satisfy the IQC defined by $\Pi$, if

$$
\int_{-\infty}^{\infty}\left[\begin{array}{c}
\hat{v}(j \omega) \\
\hat{w}(j \omega)
\end{array}\right]^{*} \Pi(j \omega)\left[\begin{array}{c}
\hat{v}(j \omega) \\
\hat{w}(j \omega)
\end{array}\right] d \omega \geq 0
$$

where absolute integrability is assumed. Here $\hat{v}(j \omega)$ and $\hat{w}(j \omega)$ represent the harmonic spectrum of the signals $v$ and $w$ at the frequency $\omega$. In principle, $\Pi: j \mathbb{R} \rightarrow \mathbb{C}$ can be any measurable Hermitian-valued function. In most applications, however, it is sufficient to use rational functions that are bounded on the imaginary axis.

A time-domain form of (4) is

$$
\int_{0}^{\infty} \sigma\left(x_{\pi}(t), v(t), w(t)\right) d t \geq 0
$$


where $\sigma$ is a quadratic form, and $x_{\pi}$ is defined by

$$
\dot{x}_{\pi}(t)=A_{\pi} x_{\pi}(t)+B_{v} v(t)+B_{w} w(t), \quad x_{\pi}(0)=0
$$

where $A_{\pi}$ is a Hurwitz matrix.

The main theorem from [9] goes as follows

Theorem 1 ([9]) Let $G \in \mathcal{R H}_{\infty}$ and let $\Delta$ be a bounded causal operator. Assume that:

i) for every $\tau \in[0,1]$, the interconnection of $G$ and $\tau \Delta$ is well-posed;

ii) for every $\tau \in[0,1]$, the IQC defined by $\Pi$ is satisfied by $\tau \Delta$;

iii) there exists $\epsilon>0$ such that

$$
\left[\begin{array}{c}
G(j \omega) \\
I
\end{array}\right]^{*} \Pi(j \omega)\left[\begin{array}{c}
G(j \omega) \\
I
\end{array}\right] \leq-\epsilon I, \quad \forall \omega \in \mathbb{R}
$$

Then the feedback interconnection of $G$ and $\Delta$ is stable.

Note that if the upper left corner, $\Pi_{11}(j \omega)$, of $\Pi$ is positive semidefinite for all $\omega \in \mathbb{R}$ then $\Delta=0$ satisfies (4). If further the lower right corner, $\Pi_{22}(j \omega)$, is negative semidefinite for all $\omega \in \mathbb{R}$, then any convex combination of $\Delta$ 's satisfying (4) also satisfies the IQC. Thus, $\Pi_{11} \geq 0$ and $\Pi_{22} \leq 0$ imply that $\tau \Delta$ satisfies (4) for $\tau \in[0,1]$ if and only if $\Delta$ does so. This simplifies assumption ii).

The search for multipliers, $\Pi$, can be carried out as a convex optimization problem by parametrizing

$$
\Pi(j \omega)=\sum_{i} x_{i} \Pi_{i}(j \omega)
$$

where $x_{i}$ are positive real parameters and $\Pi_{i}$ is a set of basis multipliers. Usually, $\Pi_{i}$ and $G$ are proper rational functions with no poles on the imaginary axis, so that we can rewrite

$$
\begin{aligned}
& {\left[\begin{array}{c}
G(j \omega) \\
I
\end{array}\right]^{*} \Pi_{i}(j \omega)\left[\begin{array}{c}
G(j \omega) \\
I
\end{array}\right]} \\
& \quad=\left[\begin{array}{c}
D+C(j \omega I-A)^{-1} B \\
I
\end{array}\right]^{*} M_{i}\left[\begin{array}{c}
D+C(j \omega I-A)^{-1} B \\
I
\end{array}\right] .
\end{aligned}
$$

In this formulation the matrices $A, B, C$ and $D$ depend on $G$ and $\Pi$, while $M_{i}$ depends on $\Pi_{i}$ only. Thus, $M$ is independent of $G$.

By applying the Kalman-Yakubovich-Popov lemma [17, 18, 12], the search for $x_{i}$, can be implemented using linear matrix inequalities (LMIs). Then (6) is equivalent to the existence of $P=P^{*}$ such that

$$
\left[\begin{array}{cc}
P A+A^{*} P & P B \\
B^{*} P & 0
\end{array}\right]+\left[\begin{array}{cc}
C & D \\
0 & I
\end{array}\right]^{*} M\left[\begin{array}{cc}
C & D \\
0 & I
\end{array}\right]<0
$$

holds, where

$$
M=\left[\begin{array}{ll}
M_{11} & M_{12} \\
M_{12}^{*} & M_{22}
\end{array}\right]=\sum_{i} x_{i} M_{i}
$$




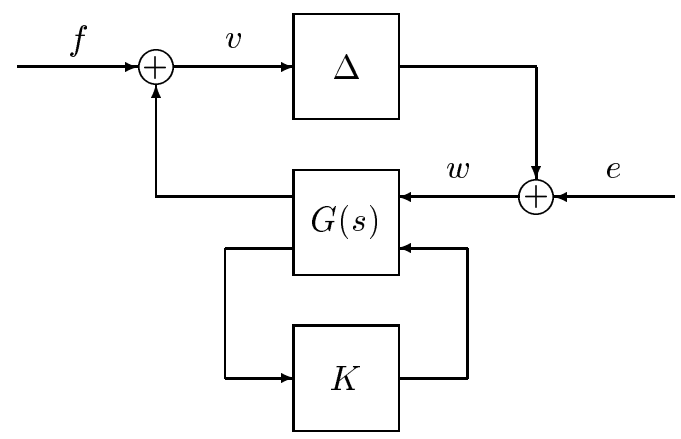

Figure 2: Feedback configuration with a controller $K$.

Note that this can also be written as

$$
\left[\begin{array}{cc}
A & B \\
C & D \\
\hline I & 0 \\
0 & I
\end{array}\right]^{*}\left[\begin{array}{cc|cc}
0 & 0 & P & 0 \\
0 & M_{11} & 0 & M_{12} \\
\hline P & 0 & 0 & 0 \\
0 & M_{12}^{*} & 0 & M_{22}
\end{array}\right]\left[\begin{array}{cc}
A & B \\
C & D \\
\hline I & 0 \\
0 & I
\end{array}\right]<0 .
$$

In a more general setting, we may also let $M$ be defined as a convex set specified by an LMI. For instance, we may add constraints such that $\Pi_{11}(j \omega)>0$ and $\Pi_{22}(j \omega)<0$, for all $\omega \in \mathbb{R}$, see $[9,7]$ for examples.

\subsection{Controller Synthesis}

In (7), $A, B, C$ and $D$ depend on $G$ and $\Pi$, while $M$ depends on $\Pi$ only, that is, $M$ is independent of $G$. We may let $G$ or, equivalently, $A, B, C$ and $D$ depend on some controller, see figure 2 . We assume that they are parametrized as a linear fractional transformation (LFT). It is no loss of generality to assume that the controller is represented as a static matrix; dynamics can be included by augmenting $G$. Thus,

$$
\left[\begin{array}{ll}
A & B \\
C & D
\end{array}\right]=\left[\begin{array}{ll}
\tilde{A} & \tilde{B} \\
\tilde{C} & \tilde{D}
\end{array}\right] \star K=\tilde{A}+\tilde{B} K(I-\tilde{D} K) \tilde{C} .
$$

If we assume that $\tilde{D}=0$, the matrices $A, B, C$ and $D$ depend affinely on $K$. If $\tilde{D} \neq 0$, we replace $K$ with

$$
K=\left[\begin{array}{cc}
0 & I \\
I & -\tilde{D}
\end{array}\right] \star \tilde{K}=\tilde{K}(I+\tilde{D} \tilde{K})^{-1}
$$

Then,

$$
\left[\begin{array}{ll}
A & B \\
C & D
\end{array}\right]=\left[\begin{array}{cc}
\tilde{A} & \tilde{B} \\
\tilde{C} & \tilde{D}
\end{array}\right] \star K=\left[\begin{array}{cc}
\tilde{A} & \tilde{B} \\
\tilde{C} & 0
\end{array}\right] \star \tilde{K}=\tilde{A}+\tilde{B} \tilde{K} \tilde{C}
$$

which depends affinely on $\tilde{K}$. The modified problem is equivalent to the original one as long as $I+\tilde{D} \tilde{K}$ is nonsingular.

Thus, we have arrived at a the following matrix inequality problem. Determine if there exists a controller, $\tilde{K}$, such that

$$
\left[\begin{array}{c}
\tilde{A}+\tilde{B} \tilde{K} \tilde{C} \\
I
\end{array}\right]^{*} \Pi\left[\begin{array}{c}
\tilde{A}+\tilde{B} \tilde{K} \tilde{C} \\
I
\end{array}\right]<0
$$


holds. If such a controller exists, find one such controller or, if possible, find the set of all controllers that satisfy (8). In this paper we will focus on the existence conditions. In order to simplify the notation, we will rewrite (8) as

$$
(A+B K C)^{*} \Pi(A+B K C)<0
$$

where

$$
A=\left[\begin{array}{c}
\tilde{A} \\
I
\end{array}\right], \quad B=\left[\begin{array}{c}
\tilde{B} \\
0
\end{array}\right], \quad C=\tilde{C}, \quad \text { and } \quad K=\tilde{K} .
$$

In both of this two formulations it is assumed that $\Pi$ has a given structure, for instance

$$
\Pi=\left[\begin{array}{cc|cc}
0 & 0 & P & 0 \\
0 & M_{11} & 0 & M_{12} \\
\hline P & 0 & 0 & 0 \\
0 & M_{12}^{*} & 0 & M_{22}
\end{array}\right]
$$

where $P=P^{*}$ and $M=M^{*}$ are convex sets.

\section{$3 \quad$ Matrix Inertia}

The conditions for having a solution to the synthesis problem will be based on the inertia of matrices. The inertia of a matrix is defined as the numbers of negative, zero and positive eigenvalues. We will denote the number of negative eigenvalues of a (square) matrix $A$ by $\nu(A)$ and the number of positive eigenvalues by $\pi(A)=\nu(-A)$. In the sequel we will only consider the inertia of hermitian matrices.

One important fact (a theorem by Sylvester and Jacobi) of the inertia of an hermitian matrix is that it is unaffected by any congruence transformation, see for instance [16]. A congruence transformation of a matrix $P=P^{*}$ is $T^{*} P T$ where $T$ is any nonsingular (square) matrix. Thus, $\nu(P)=\nu\left(T^{*} P T\right)$.

Lemma 1 The truncation $\Pi_{11}$ of a hermitian matrix $\Pi=\left[\begin{array}{ll}\Pi_{11} & \Pi_{12} \\ \Pi_{12}^{*} & \Pi_{22}\end{array}\right]$ satisfies $\nu(\Pi) \geq \nu\left(\Pi_{11}\right)$.

Proof: First assume that $\Pi_{11}$ is nonsingular. Then

$$
\left[\begin{array}{cc}
I & -\Pi_{11}^{-1} \Pi_{12} \\
0 & I
\end{array}\right]^{*}\left[\begin{array}{ll}
\Pi_{11} & \Pi_{12} \\
\Pi_{12}^{*} & \Pi_{22}
\end{array}\right]\left[\begin{array}{cc}
I & -\Pi_{11}^{-1} \Pi_{12} \\
0 & I
\end{array}\right]=\left[\begin{array}{cc}
\Pi_{11} & 0 \\
0 & \Pi_{22}-\Pi_{12}^{*} \Pi_{11}^{-1} \Pi_{12}
\end{array}\right],
$$

and consequently $\nu(\Pi)=\nu\left(\Pi_{11}\right)+\nu\left(\Pi_{22}-\Pi_{12}^{*} \Pi_{11}^{-1} \Pi_{12}\right) \geq \nu\left(\Pi_{11}\right)$.

If $\Pi_{11}$ is singular then we can modify the problem without affecting $\nu(\Pi)$ nor $\nu\left(\Pi_{11}\right)$, by adding $\varepsilon I$ to $\Pi_{11}$, where $\varepsilon>0$ is sufficiently small. For a given $\Pi$, we can choose $\varepsilon$ to be less than the minimum of the absolute values of the negative eigenvalues of $\Pi_{11}$ and $\Pi$.

Note that the trick of modifying a singular matrix, say $\Pi$, without modifying $\nu(\Pi)$ will be used for derivation of some results in the sequel. Such a modification 
does affect the inertia since it modifies the number of zero eigenvalues. However, since we here only consider the number of negative (or positive) eigenvalues this operation is legal.

The following lemma connects a certain structure of inertia conditions to LMIs.

Lemma 2 Let $X \in \mathbb{R}^{n \times n}$. Then

$$
\nu\left(\left[\begin{array}{cc}
0 & B^{*} \\
B & X
\end{array}\right]\right) \geq n
$$

if and only if $B^{\perp} X B^{\perp *}<0$.

Proof: For any sufficiently small $\varepsilon>0,(10)$ is equivalent to

$$
\nu\left(\left[\begin{array}{cc}
\varepsilon I_{m} & B^{*} \\
B & X
\end{array}\right]\right)=\nu\left(\left[\begin{array}{cc}
\varepsilon I_{m} & 0 \\
0 & X-\varepsilon^{-1} B B^{*}
\end{array}\right]\right)=\nu\left(X-\varepsilon^{-1} B B^{*}\right)=n \geq n,
$$

which in turn is equivalent to $X<\varepsilon^{-1} B B^{*}$ for any sufficiently small $\varepsilon>0$, or equivalently, using Finsler's theorem, see for instance [11, 8], $B^{\perp} X B^{\perp *}<0$.

Note that in this case (10) is an equality, since $\nu(X) \leq n$ for any matrix $X=$ $X^{*} \in \mathbb{R}^{n \times n}$.

\subsection{Reformulations}

Conditions on the inertia of a matrix can be seen as an extension to the linear matrix inequalities (LMIs). For instance, if $P=P^{*} \in \mathbb{R}^{n \times n}$. Then $\nu(P) \geq n$, or $\nu(P)=n$, is equivalent to $P<0$. Other conditions on the inertia can be translated into LMIs with rank constraints.

Lemma 3 Let $P=P^{*} \in \mathbb{R}^{(n+m) \times(n+m)}$. The following three statements are equivalent:

(i) $\nu(P) \geq n$;

(ii) There exists a $Q=Q^{*} \geq 0$ with $\operatorname{rank} Q \leq m$ such that $P<Q$;

(iii) There exists a $U \in \mathbb{R}^{(n+m) \times n}$ such that $U^{*} P U<0$.

Proof: (i) $\Rightarrow$ (ii) Diagonalizing $P$ using a congruent transformation yields a matrix with its eigenvalues along its diagonal. It is clear that there are no more than $m$ non-negative eigenvalues and there are at least $n$ negative eigenvalues. Thus, it is clear that we can choose a $Q \geq 0$ such that $\operatorname{rank} Q \leq m$.

(ii) $\Rightarrow$ (iii) Choose $U$ as a full rank matrix spanning the nullspace of $Q$. Note that $U$ has at least $n$ columns; truncate it, if necessary, to exactly $n$ columns. Then, $U^{*}(P-Q) U=U^{*} P U-0<0$.

(iii) $\Rightarrow$ (i) It is clear that we can find a full rank matrix $V$ such that $[U V]$ becomes nonsingular. Using lemma 1 it follows that $\nu(P) \geq \nu\left(U^{*} P U\right)=n$.

LMIs with rank constraints also emerge in synthesis of reduced-order controllers and model reduction. In general, these problems are hard to solve, since they are not convex. Several methods have been proposed for this class of problems, for instance projection methods [6], inversion of analytic centering [2], and bilinear matrix inequalities (BMIs) [5, 4, 3, 13]. 


\subsection{Solving Inertia Inequalities}

We will here briefly discuss how problems with inertia constraints can be solved numerically. We assume that there are constraints on the form $\nu(F(x)) \geq n$ and $C(x)>0$, where $F$ and $C$ are affine functions of $x$. We may maximize or minimize a linear combination of $x$, that is $c^{T} x$, subject to these constraints.

Such an optimization could be based on (local) optimization subject to a barrier function. One choice is to use $\Phi(x)=\log \operatorname{det} C(x)+\log |\operatorname{det} F(x)|$, which goes to infinity as the constraints are violated.

This is similar to the barrier function used in algorithms for solving linear matrix inequalities, see for instance [1]. The analytic center and the analytic path both play important role in these algorithms. Both are the minimizers of $\Phi(x)$ subject to $F(x)>0$ and $C(x)>0$, in the latter also subject to $c^{T} x=\gamma$.

It is possible to compute the the minimum of $\Phi(x)$ even if $F(x)$ is not positive definite. However, since the convexity is lost, $\Phi(x)$ may have several local minima, which have to be searched for. Also, the barrier function may divide the parameter space into several non-connected, non-convex regions, with the same number of negative eigenvalues, $\nu(F(x))$.

As the size of $F$ increases the number of local minima is likely to increase and the complexity of the problem increases as well. In [2] it is shown that an LMI problem with rank constraints is NP-hard. Consequently, according to lemma 3, problems with inertia constraints are NP-hard as well.

Despite this fact, numerical algorithm searching for local minima as described above, could work well in many applications, especially if the search starts from an well-educated guess.

\section{Synthesis}

We will here study the general synthesis problem: what are the conditions for the existence of $K \in \mathbb{R}^{m \times p}$ such that

$$
(A+B K C)^{*} \Pi(A+B K C)<0
$$

holds. We start by looking at special case, which provides a simpler problem.

Lemma 4 There exists a $K \in \mathbb{R}^{m \times p}$ such that

$$
\left[\begin{array}{c}
I \\
K
\end{array}\right]^{*} \Pi\left[\begin{array}{c}
I \\
K
\end{array}\right]<0
$$

if and only if $\nu(\Pi) \geq p$.

Proof: $(\Rightarrow)$ Applying lemma 1 to

$$
P=\left[\begin{array}{cc}
I & 0 \\
K & I
\end{array}\right]^{*} \Pi\left[\begin{array}{cc}
I & 0 \\
K & I
\end{array}\right]
$$

and using (11), yields $\nu(\Pi)=\nu(P) \geq \nu\left(P_{11}\right)=p$.

$$
(\Leftarrow) \text { Let } U=\left[\begin{array}{l}
U_{1} \\
U_{2}
\end{array}\right] \text { be a matrix spanning the eigenspace corresponding to }
$$

the negative eigenvalues of $\Pi$. If $U_{1} \in \mathbb{R}^{p \times p}$ is non-singular then $K=U_{2} U_{1}^{-1}$ 
satisfies (11). If $U_{1}$ is singular add $\varepsilon I$ to it, where $\varepsilon>0$ is a sufficiently small number such that

$$
\left[\begin{array}{c}
U_{1}+\varepsilon I \\
U_{2}
\end{array}\right]^{*} \Pi\left[\begin{array}{c}
U_{1}+\varepsilon I \\
U_{2}
\end{array}\right]<0
$$

still holds and use $K=U_{2}\left(U_{1}+\varepsilon I\right)^{-1}$.

\subsection{Main Theorem}

We are now ready to state and prove the following new theorem.

Theorem 2 Let $A \in \mathbb{R}^{k \times n}, B \in \mathbb{R}^{k \times m}$ and $C \in \mathbb{R}^{p \times n}$. There exists a $K \in$ $\mathbb{R}^{m \times p}$ such that

$$
(A+B K C)^{*} \Pi(A+B K C)<0
$$

holds if and only if

$$
\begin{aligned}
& C^{* \perp} A^{*} \Pi A C^{* \perp *}<0 \\
& \nu\left(\left[\begin{array}{ll}
A & B
\end{array}\right]^{*} \Pi\left[\begin{array}{ll}
A & B
\end{array}\right]\right) \geq n .
\end{aligned}
$$

Proof: It is clear that (13a) is a necessary condition. Without loss of generality we may assume that $C$ is full row rank. If not, we replace $C$ in the original problem by a full rank matrix with the same nullspace and modify the size of $K$ accordingly, so that the set of matrices generated by $K C$ is unaffected.

By pre-multiplying (12) by $C^{* \perp}$ and post-multiplying by its transpose, we infer that (13a) is a necessary condition. We absorb the dependency on $A$ and $B$ onto $\Pi$ by rewriting (12) as

$$
\begin{aligned}
& (A+B K C)^{*} \Pi(A+B K C) \\
& =\left[\begin{array}{c}
I \\
K C
\end{array}\right]^{*}\left[\begin{array}{ll}
A & B
\end{array}\right]^{*} \Pi\left[\begin{array}{ll}
A & B
\end{array}\right]\left[\begin{array}{c}
I \\
K C
\end{array}\right] \\
& =\left[\begin{array}{c}
I \\
K C
\end{array}\right]^{*} P\left[\begin{array}{c}
I \\
K C
\end{array}\right]<0
\end{aligned}
$$

where $P=\left[\begin{array}{ll}A & B\end{array}\right]^{*} \Pi\left[\begin{array}{ll}A & B\end{array}\right]$.

We transform (12) into an equivalent problem by performing a congruence transformation using $\left[C^{* \dagger *} C^{* \perp *}\right]$, such that $C\left[C^{* \dagger *} C^{* \perp *}\right]=\left[\begin{array}{ll}I_{p} & 0\end{array}\right]$. Denote by

$$
\tilde{A}_{1}=\left[\begin{array}{c}
C^{* \dagger *} \\
0
\end{array}\right] \quad \tilde{A}_{2}=\left[\begin{array}{c}
C^{* \perp *} \\
0
\end{array}\right] \quad \tilde{B}=\left[\begin{array}{l}
0 \\
I
\end{array}\right] .
$$

The inequality (12) is equivalent to

$$
\begin{aligned}
& {\left[\begin{array}{ll}
\tilde{A}_{1}+\tilde{B} K & \tilde{A}_{2}
\end{array}\right]^{*} P\left[\begin{array}{ll}
\tilde{A}_{1}+\tilde{B} K & \tilde{A}_{2}
\end{array}\right]} \\
& =\left[\begin{array}{cc}
\left(\tilde{A}_{1}+\tilde{B} K\right)^{*} P\left(\tilde{A}_{1}+\tilde{B} K\right) & \left(\tilde{A}_{1}+\tilde{B} K\right)^{*} P \tilde{A}_{2} \\
\tilde{A}_{2}^{*} P\left(\tilde{A}_{1}+\tilde{B} K\right) & \tilde{A}_{2}^{*} P \tilde{A}_{2}
\end{array}\right]<0 .
\end{aligned}
$$


Since (13a) or, equivalently, $\tilde{A}_{2}^{*} P \tilde{A}_{2}<0$ holds, we rewrite (14) using the Schur complement as

$$
\begin{aligned}
& \left(\tilde{A}_{1}+\tilde{B} K\right)^{*} P\left(\tilde{A}_{1}+\tilde{B} K\right)-\left(\tilde{A}_{1}+\tilde{B} K\right)^{*} P \tilde{A}_{2}\left(\tilde{A}_{2}^{*} P \tilde{A}_{2}\right)^{-1} \tilde{A}_{2}^{*} P\left(\tilde{A}_{1}+\tilde{B} K\right) \\
& =\left(\tilde{A}_{1}+\tilde{B} K\right)^{*} Q\left(\tilde{A}_{1}+\tilde{B} K\right)^{*}<0
\end{aligned}
$$

where $Q=P-P \tilde{A}_{2}\left(\tilde{A}_{2}^{*} P \tilde{A}_{2}\right)^{-1} \tilde{A}_{2}^{*} P$. Next, we transform (15) into

$$
\begin{aligned}
\left(\tilde{A}_{1}+\tilde{B} K\right)^{*} Q\left(\tilde{A}_{1}+\tilde{B} K\right)^{*} & =\left[\begin{array}{c}
C^{* \dagger *} \\
K
\end{array}\right]^{*} Q\left[\begin{array}{c}
C^{* \dagger *} \\
K
\end{array}\right] \\
& =\left[\begin{array}{c}
I \\
K
\end{array}\right]^{*} D^{*} Q D\left[\begin{array}{c}
I \\
K
\end{array}\right]<0
\end{aligned}
$$

where

$$
D=\left[\begin{array}{cc}
C^{* \dagger *} & 0 \\
0 & I_{m}
\end{array}\right] .
$$

Using lemma 4, we infer that (16) has a solution $K$ if and only if $\nu\left(D^{*} Q D\right) \geq p$. Observing that $C^{* \perp}$ has $n-p$ rows, and consequently $n-p=\nu\left(\tilde{A}_{2}^{*} P \tilde{A}_{2}\right)=$ $\nu\left(C^{* \perp} A^{*} \Pi A C^{* \perp *}\right)$, it follows that

$$
\begin{aligned}
n=p+(n-p) & \leq \nu\left(D^{*} Q D\right)+\nu\left(\tilde{A}_{2}^{*} P \tilde{A}_{2}\right) \\
& =\nu\left(\left[\begin{array}{cc}
D^{*}\left(P-P \tilde{A}_{2}\left(\tilde{A}_{2}^{*} P \tilde{A}_{2}\right)^{-1} \tilde{A}_{2}^{*} P\right) D & 0 \\
0 & \tilde{A}_{2}^{*} P \tilde{A}_{2}
\end{array}\right]\right) \\
& =\nu\left(\left[\begin{array}{cc}
\tilde{A}_{2}^{*} P \tilde{A}_{2} & \tilde{A}_{2}^{*} P D \\
D^{*} P \tilde{A}_{2} & D^{*} P D
\end{array}\right]\right) \\
& =\nu\left(\left[\begin{array}{cc}
\tilde{A}_{2} & D
\end{array}\right]^{*} P\left[\begin{array}{cc}
\tilde{A}_{2} & D
\end{array}\right]\right) \\
& =\nu\left(\left[\begin{array}{ccc}
C^{* \perp *} & C^{* \dagger *} & 0 \\
0 & 0 & I
\end{array}\right]^{*} P\left[\begin{array}{ccc}
C^{* \perp *} & C^{* \dagger *} & 0 \\
0 & 0 & I
\end{array}\right]\right) \\
& =\nu(P)=\nu\left(\left[\begin{array}{ll}
A & B
\end{array}\right]^{*} \Pi\left[\begin{array}{cc}
A & B
\end{array}\right]\right),
\end{aligned}
$$

where we have used a congruence transformation similar to the one in (9).

Note that the condition (13a), including $C$, is convex in $\Pi$, while the condition (13b), including $B$, is in general not convex.

Condition (13b) tells us that the number of negative eigenvalues in

$$
\left[\begin{array}{ll}
A & B
\end{array}\right]^{*} \Pi\left[\begin{array}{ll}
A & B
\end{array}\right] \text {, }
$$

must be greater than or equal to the number of rows in $A$.

We can also reformulate the inertia condition (13b) using lemma (3), as the existence of a full row rank matrix matrix $U \in \mathbb{R}^{(n+m) \times p}$ such that

$$
U^{*}\left[\begin{array}{ll}
A & B
\end{array}\right]^{*} \Pi\left[\begin{array}{ll}
A & B
\end{array}\right] U<0 .
$$

This can be interpreted as an LMI where $U$ selects the appropriate subspace from $\left[\begin{array}{ll}A & B\end{array}\right]$. Note that this reformulation is not convex since $U$ is not given, but must be searched for. The space spanned by $U$ must at least contain the space spanned by $\left[\begin{array}{ll}C^{* \perp} & 0\end{array}\right]^{*}$. 


\subsection{The Standard IQC Synthesis Case}

We will now reconsider the standard IQC synthesis problem (8), for which we state and prove the following new theorem.

Theorem 3 Let $A \in \mathbb{R}^{k \times n}, B \in \mathbb{R}^{k \times m}$ and $C \in \mathbb{R}^{p \times n}$. Assume that $\Pi=\Pi^{*} \in$ $\mathbb{R}^{(k+n) \times(k+n)}$ is non-singular and has $k$ positive and $n$ negative eigenvalues. Then there exists a $K \in \mathbb{R}^{m \times p}$ such that

$$
\left[\begin{array}{c}
A+B K C \\
I
\end{array}\right]^{*} \Pi\left[\begin{array}{c}
A+B K C \\
I
\end{array}\right]<0
$$

if and only if

$$
\begin{aligned}
& C^{* \perp}\left[\begin{array}{c}
A \\
I
\end{array}\right]^{*} \Pi\left[\begin{array}{c}
A \\
I
\end{array}\right] C^{* \perp *}<0, \\
& B^{\perp}\left[\begin{array}{c}
I \\
-A^{*}
\end{array}\right]^{*} \Pi^{-1}\left[\begin{array}{c}
I \\
-A^{*}
\end{array}\right] B^{\perp *}>0 .
\end{aligned}
$$

Proof: We apply theorem 2: the first condition (13a) gives (18a), and the second condition (13b) becomes

$$
\nu\left(\left[\begin{array}{cc}
A & B \\
I & 0
\end{array}\right]^{*} \Pi\left[\begin{array}{cc}
A & B \\
I & 0
\end{array}\right]\right) \geq n .
$$

Using a congruence transformation, (19) is equivalent to

$$
\begin{aligned}
& n+k \leq \nu\left(\left[\begin{array}{cc}
A & B \\
I & 0
\end{array}\right]^{*} \Pi\left[\begin{array}{cc}
A & B \\
I & 0
\end{array}\right]\right)+\pi\left(\Pi^{-1}\right) \\
& =\nu\left(\left[\begin{array}{cc}
{\left[\begin{array}{cc}
A & B \\
I & 0
\end{array}\right]^{*} \Pi\left[\begin{array}{cc}
A & B \\
I & 0
\end{array}\right]} & 0 \\
\hline 0 & -\Pi^{-1}
\end{array}\right]\right) \\
& =\nu\left(\left[\begin{array}{cc|cc}
0 & 0 & A^{*} & I \\
0 & 0 & B^{*} & 0 \\
\hline A & B & -\Pi^{-1} \\
I & 0 &
\end{array}\right]\right) \text {. }
\end{aligned}
$$

We next use lemma 2, with

$$
B^{\perp}\left[\begin{array}{ll}
I & -A
\end{array}\right]=\left[\begin{array}{cc}
A & B \\
I & 0
\end{array}\right]^{\perp},
$$

which yields (18b).

The inertia assumption on $\Pi$ can relaxed to $\pi(\Pi) \geq k$. Since (19) must hold, we infer that $\nu(\Pi)=n$ and $\pi(\Pi)=k$.

In previous synthesis results using IQCs, the assumption on

$$
\Pi=\left[\begin{array}{cc}
P & \Gamma \\
\Gamma^{*} & -Q
\end{array}\right],
$$

is that $P$ and $Q$ are both positive (semi-)definite, see $[10,14,15]$. In theorem 3 the only assumption on $\Pi$ is its inertia, which is weaker than in previous results.

In many applications we may apply the following lemma. 
Lemma 5 Let

$$
\Pi=\left[\begin{array}{cc}
P & \Gamma \\
\Gamma^{*} & -Q
\end{array}\right]
$$

be a nonsingular matrix where $P \in \mathbb{R}^{k \times k}, Q \in \mathbb{R}^{n \times n}$ and $P, Q \geq 0$. Then $\nu(\Pi)=k$ and $\pi(\Pi)=n$.

Proof: If $P$ is nonsingular, that is $P>0$, then

$$
\Pi=\left[\begin{array}{cc}
P & \Gamma \\
\Gamma^{*} & -Q
\end{array}\right] \sim\left[\begin{array}{cc}
P & 0 \\
0 & -Q-\Gamma^{*} P^{-1} \Gamma
\end{array}\right]
$$

where $\sim$ denotes similarity by a congruence transformation. Consequently, $\nu(\Pi)=k$ and $\pi(\Pi)=\nu\left(Q+\Gamma^{*} P^{-1} \Gamma\right)=k$, since $\Pi$ is nonsingular and $Q \geq 0$. If $P \geq 0$ is singular then we can modify it by adding $\varepsilon I_{n}$, such that the inertia of $\Pi$ is unchanged ( $\Pi$ is nonsingular), where $\varepsilon>0$ is a sufficiently small real number.

However, in some applications the assumption that $P$ and $Q$ are positive semidefinite is too conservative, see for instance [7]

\subsection{An example - Static feedback}

A simple problem concerns the stabilization of a linear time-invariant system, $G(s)=C(s I-A)^{-1} B$, using static feedback, $K$. The system with static feedback is strictly stable if

$$
\left[\begin{array}{c}
A+B K C \\
I
\end{array}\right]^{*}\left[\begin{array}{ll}
0 & P \\
P & 0
\end{array}\right]\left[\begin{array}{c}
A+B K C \\
I
\end{array}\right]<0,
$$

holds for some $P=P^{*}>0$.

Applying theorem 2, the existence of a $K$ for a given $P$ is equivalent to

$$
\begin{aligned}
& C^{* \perp}\left(A^{*} P+P A\right) C^{* \perp *}<0 \\
& \nu\left(\left[\begin{array}{cc}
A & B \\
I & 0
\end{array}\right]^{*}\left[\begin{array}{cc}
0 & P \\
P & 0
\end{array}\right]\left[\begin{array}{cc}
A & B \\
I & 0
\end{array}\right]\right) \geq n
\end{aligned}
$$

where $n$ is the dimension of $A$ and $P$, that is the number of states. The last condition can be rewritten as

$$
\nu\left(\left[\begin{array}{cc}
A^{*} P+P A & P B \\
B^{*} P & 0
\end{array}\right]^{*}\right) \geq n .
$$

For first and second order systems, the solution set of $P$ is convex. For thirdorder systems this is generally not true.

When $B$ is a unit or a non-singular matrix, that is the full control, the matrix

$$
\left[\begin{array}{cc}
A & B \\
I & 0
\end{array}\right]
$$

is non-singular and can be considered as a congruence transformation in (20). Thus, the number of negative eigenvalues is equal to the number of negative eigenvalues of

$$
\left[\begin{array}{ll}
0 & P \\
P & 0
\end{array}\right]
$$


which is $n$ if $P$ is nonsingular. Thus (20) is always satisfied, and the only remaining condition for the existence of a stabilizing controller is

$$
C^{* \perp}\left(A^{*} P+P A\right) C^{* \perp *}<0,
$$

which is convex in $P$. The full control problem is dual to the more common full information problem, see for instance [19].

\section{Conclusions}

Synthesis based on integral quadratic constraints (IQC) can be expressed as solving a quadratic inequality involving a parametrization of the controller. Necessary and sufficient conditions for the existence of a controller have been derived. The conditions comprise a linear matrix inequality (LMI) and a matrix inertia constraint. In general the matrix inertia condition is not convex and the problem becomes numerically hard since several local minima must be searched for and inspected.

The inertia constraint can be seen as an alternative to LMIs with rank constraints and to bilinear matrix inequalities (BMIs). It is hoped that this new formulation could lead to better insight into the synthesis problem in order to better understand the problem and its complexity.

\section{References}

[1] S. Boyd, L. El Ghaoui, E. Feron, and V. Balakrishnan. Linear Matrix Inequalities in System and Control Theory. SIAM Studies in Applied Mathematics. SIAM, 1994.

[2] J. David. Algorithms for Analysis and Design of Robust Controllers. PhD thesis, Dept. of Electrical Engineering, K. U. Leuven, Leuven, Belgium, 1994.

[3] K. Goh, J. H. Ly, L. Turan, and M. Safonov. $\mu / K_{m}$-synthesis via bilinear matrix inequalities. In Proceedings of the 33rd Conference on Decision and Control, volume 3, pages 2032-2037, Lake Buena Vista, Florida, December 1994.

[4] K. Goh, M. Safonov, and G. Papavassilopoulos. A global optimization approach for the BMI problem. In Proceedings of the 33rd Conference on Decision and Control, volume 3, pages 2009-2014, Lake Buena Vista, Florida, December 1994.

[5] K. Goh, L. Turan, M. Safonov, G. Papavassilopoulos, and J. Ly. Biaffine matrix inequality properties and computational methods. In Proceedings of the American Control Conference, volume 1, pages 850-855, Baltimore, Maryland, June 1994.

[6] K. Grigoriadis and R. Skelton. Fixed-order control design for LMI control problems using alternating projection methods. In Proceedings of the 33rd Conference on Decision and Control, volume 3, pages 2003-2008, Lake Buena Vista, Florida, December 1994. 
[7] A. Helmersson. An IQC-based stability criterion for systems with slowly varying parameters. Technical Report LiTH-ISY-R-1979, Linköping University, Linköping, Sweden, 1997. Submitted to the ACC 1998 conference.

[8] T. Iwasaki and R. E. Skelton. All controllers for the general $H_{\infty}$ control problem: LMI existence conditions and state space formulas. Automatica, 30:1307-1317, August 1994.

[9] A. Megretski and A. Rantzer. System analysis via integral quadratic constraints. IEEE Transactions on Automatic Control, 42(6):819-830, June 1997.

[10] R. Njio, C. Scherer, and S. Bennani. Application of LPV control with full block scalings for a high performance flight control system. In Selected Topics in Identification, Modelling and Control, pages 113-120. Delft University Press, Delft, Netherlands, December 1996.

[11] I. R. Petersen and C. V. Hollot. A Riccati equation approach to the stabilization of uncertain linear systems. Automatica, 22:397-411, January 1986.

[12] A. Rantzer. A note on the Kalman-Yacubovich-Popov lemma. In Proceedings of the 3rd European Control Conference, volume 3, part 1, pages 1792-1795, Rome, Italy, September 1995.

[13] M. Safonov, K. Goh, and J. Ly. Control system synthesis via bilinear matrix inequalities. In Proceedings of the American Control Conference, volume 1, pages 45-49, Baltimore, Maryland, June 1994.

[14] G. Scorletti and L. El Ghaoui. Improved linear matrix inequalities conditions for gain-scheduling. In IEEE Proceedings of the 31st Conference on Decision and Control, volume 4, pages 3626-3631, New Orleans, Louisiana, December 1995.

[15] G. Scorletti and L. El Ghaoui. Imporved LMI conditions for gain scheduling and related control problems. International Journal of Robust and Nonlinear Control, 1997. Accepted for publication.

[16] G. W. Stewart and J. Sun. Matrix Perturbation Theory. Computer Science and Scientific Computing. Academic Press, 1990.

[17] J. C. Willems. The Analysis of Feedback Systems. MIT Press, Cambridge, MA, 1971.

[18] V. A. Yakubovich. A frequency theorem for the case in which the state and control spaces are Hilbert spaces with an application to some problems of optimal controls - Part I-II. Sibirskii Mat. Zh., 15(3):639-668, 1974. English translation in Siberian Math. J.

[19] K. Zhou, J. C. Doyle, and K. Glover. Robust and Optimal Control. Prentice Hall, 1995. 